Was aus dem Beispiel der amerikanischen Umweltbehörde gelernt werden kann

\section{Verwaltungsreform und Nachhaltigkeitsziele}

\section{Die Reform der Verwaltung ist in Deutschland erklärtes politisches Ziel. Die Überlegungen zur Effizienzsteigerung der Verwaltungen stammen vor allem aus den angelsächsischen Ländern. Hier liegen Erfahrungen vor, die gerade für die Verfolgung von Nachhaltigkeitszielen fruchtbar gemacht werden können und deutschen Aktivitäten als Vorbild dienen.}

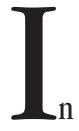

\section{Felicitas Kubala und Ulrich Petschow}

n jüngerer Zeit ist in den USA ein neuer Versuch der Verwaltungsreform mit dem Government Performance and Results Act (GPRA) aus dem Jahre 1993 gestartet worden. Dieser fordert von den Bundesbehörden - und damit auch der Umweltbehörde Environmental Protection Agency (EPA) -, dass ab dem Jahre 1999 jährliche Performance-Pläne zu erstellen sind. Diese sollen zum einen die Programmaktivitäten der jeweiligen Behörden abdecken und zum anderen die Verbindung zum Budget herstellen. Das zentrale Ziel dieser Bemühungen ist, das Verwaltungshandeln auf die Ergebnisse (den Outcome) und nicht auf die Aktivitäten an sich (den Output) zu orientieren. Die Reformbemühungen haben damit eine neuen und für den Umweltzusammenhang interessanten Ansatzpunkt.

Dieser Grundansatz ist auch von der Umweltverwaltung in Schleswig-Holstein aufgegriffen worden. Es wurde ein langfristiger Nachhaltigkeitsplan entwickelt. Zur Überprüfung der gewählten Indikatoren hinsichtlich der Eignung zur Messung der Nachhaltigkeitsziele, der Datenverfügbarkeit sowie der Verknüpfung dieses Indikatorensystems mit der Kosten- und Leistungsrechnung (KLR) und dem Controlling wurde ein Gutachtenauftrag an das Ökologie-Zentrum Kiel und das IÖW vergeben (1). Im Folgenden werden am Beispiel der amerikanischen Erfahrungen die Vorgehensweise und einige ihrer Probleme dargestellt.

\section{- Das amerikanische Vorgehen}

Der GPRA und die dort geforderte Ausrichtung auf den Outcome führt in einem ersten Schritt zu einer Definition der Ziele der jeweiligen Verwaltungseinheiten. Typisierend lässt sich das Vorgehen wie folgt darstellen (vgl. Abb. 1):

- Entwicklung einer strategischen Zielplanung,

- Ableitung von jährlichen Zielen,
Identifikation der Performance und damit eine Abschätzung inwieweit die eingeleiteten Maßnahmen zur Zielerreichung beigetragen haben, sowie

- die Berichterstattung im Hinblick auf den Fortschritt der Zielerreichung.

Damit geht es im Grundsatz darum, Ziele, Mitteleinsatz und Ergebnisse im Rahmen einer Kostenund Leistungsrechnung und eines EDV gestützten Controlling in einen engen Zusammenhang zu bringen. Einige Anforderungen, die sich aus dem GPRA ergeben, sind:

- die Budgets müssen mit dem angestrebten Outcome und den strategischen Zielen (goals) in Übereinstimmung stehen;

- der strategische Plan soll eine Beschreibung der Prozesse, Technologien, der Arbeit, des eingesetzten Kapitals und anderer Ressourcen, die zur Umsetzung der Ziele erforderlich sind, beinhalten;

- die exogenen Faktoren, die die zu erreichenden Ziele beeinflussen können, müssen identifiziert werden;

- der Ressourceneinsatz soll direkt auf die Ziele ausgerichtet sein, damit wird der Budgetierungsprozess auf den Outcome orientiert;

- ein jährlicher Performance-Plan soll erstellt werden, der die Performance-Maßstäbe und die erreichten Ziele jedes Programms bzw. jeder Aktivität enthält;

- das jährliche Budget basiert auf den jährlichen Plänen, damit werden die langfristigen Ziele und der kurzfristige Planungs- und Budgetierungsprozess miteinander verbunden. Die Orientierung am Outcome hat erhebliche Aus- wirkungen auf die Wahl der Indikatoren, die Aussagen über die Ergebnisse ermöglichen müssen und eben nicht auf der Ebene der Aktivitätsindikatoren (output) stehen bleiben sollen.

\section{Umsetzung durch die EPA}

Die EPA hat dementsprechend eine langfristige Strategie entwickelt, die sich aus zehn strategischen Zielen zusammensetzt (2). Zudem wurde die Verknüpfung zur Kosten- und Leistungsrechnung und dem Controlling hergestellt, sodass es nunmehr möglich ist, die Kosten und die Umsetzungsfortschritte Ziel für Ziel zu erfassen.

Abgeleitet aus der langfristigen Strategie (,,mission") der EPA wurde ein strategischer Fünfjahresplan entwickelt. Dieser wird wiederum in einzelne Jahrespläne aufgesplittet, in denen (Kurzfrist-) Ziele festgelegt werden, die letztlich zur Zielerreichung des Fünfjahresplans (bzw. auch des strategischen Langfristzieles) führen sollen. Die Jahrespläne werden jeweils evaluiert, inwieweit die gestellten Ziele erreicht worden sind oder ob es Abweichungen gegeben hat, die gegebenenfalls zu Anpassungsmaßnahmen führen müssen.

Die Umsetzung der Vorgaben des GPRA in die Praxis einer Umweltbehörde hat einige zentrale Schwierigkeiten deutlich gemacht. Einige Aspekte sollen in der Folge kurz dargestellt werden:

\section{Zentrale Probleme}

Informationsverfügbarkeit: Eine zentrale Herausforderung ist die Entwicklung und Bereitstellung geeigneter Indikatoren und Indikatorensysteme im Hinblick auf die Performance und die zuzuordnenden Kosten. Positiv gewendet hat die neue Herangehensweise dazu geführt, dass vor allem das Problem der Verfügbarkeit konsistenter

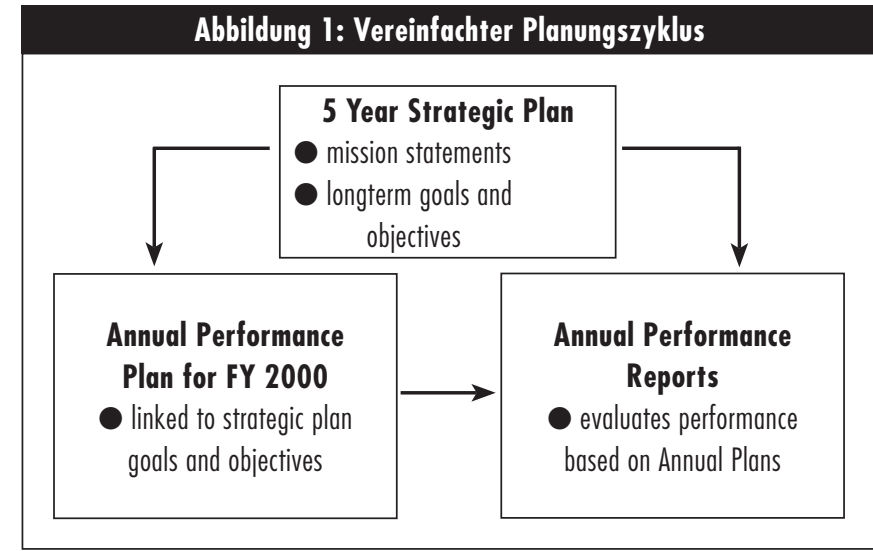

Quelle: EPA: Managing for Results. Putting the Pieces Together. EPA 1998 
Informationen für die Entscheidungsfindung angegangen wurde, allerdings von einer Lösung noch entfernt ist.

- Output/Outcome: Ein weiterer Problembereich ist die Vorgabe der Orientierung auf Wirkungen, die im Gegensatz zur Messung des Output, also eher einer Aktivitätsmessung, deutlich komplexer ist. Die exakte Messung der Beiträge von Programm-Aktivitäten zum Programm-Outcome ist eine kontinuierliche Herausforderung, da es eine

\section{politische ökologie ${ }^{a-z}$}

Wählen Sie aus unserem Gesamtverzeichnis

pö 51 Zukunftsaufgabe Umweltbildung - Auf der Suche nach neuen Perspektiven

pö 52 Geduldsspiel Nachhaltigkeit -Agenda 21 als Leitfaden für das nächste Jahrhundert

pö 53 Wechsel ohne Deckung - Über den Zusammenhang von Geld und Umwelt

pö 54 Nachhaltiges Arbeiten - Ein Weg aus der Beschäftigungs- und Umweltkrise?

pö 55 Gratwanderung - zwischen Autarkie und Globalisierung: Die Alpen als Vorreiter für ein regionales Wirtschaften

pö 56 Endspurt - Die Ökologische Steuerreform vor dem Durchbruch?

pö 57/58 Von der Zeitnot zum Zeitwohlstand - Auf der Suche nach den rechten Zeitmaßen

pö 59 Wa(h)re Wildnis - Reise Special '99

pö 60. Risiko

pö 61 Wechselstrom - Anleitung für eine neue Energiepolitik

pö 62. Stoffwechsel - Naturstrategien als Vorbild für Nachhaltigkeit

pö 63/64 Nachhaltigkeit öffne dich! Kommunikation eines Leitbildes inklusive Literatur Spezial 2000

pö 65 e_cotopia - von ökologischen visionen zu virtuellen realitäten

(Einzelhefte 19,80 DM, Doppelausgaben 24,80)

Sonderhefte

pö Sonderheft 8 Zeit-Fraß - Zur Ökologie der Zeit in Landwirtschaft und Ernährung

pö Sonderheft 9 Handwerk als Schlüssel für eine zukunftsfähige Wirtschaft

pö Sonderheft 10 Bodenlos - Zum nachhaltigen Umgang mit Böden

pö Sonderheft 11 Wege aus der Wachstumsfalle Perspektiven einer zukunftsfähigen Wirtschafts- und Lebensweise

pö Sonderheft 12 Schnittmenge Mensch - Bildung für nachhaltige Entwicklung als neue Lernkultur

\section{(Sonderhefte zum Preis von je 19,80 DM)}

PÖ-Reise-Special 2000 - Exponierte Zukunft 12,80 DM PÖ-Literatur Special - Umweltbücher 2000 12,80 DM

Abo für sechs Ausgaben: DM 98,00, (Institutionen DM 148,-), ermäßigt: DM 78,00 (ohne Versandkosten) Eine Übersicht aller noch erhältlichen Ausgaben finden Sie auch auf unserer Internetseite: www.oekom.de
Vielzahl von Faktoren gibt, die außerhalb der Kontrolle der Programm-Manager liegt und die den Outcome wesentlich mitbestimmen.

- Kooperationen: Aus dem letztgenannten Punkt ergibt sich auch, dass Umweltwirkungen Produkte eines weiten Netzwerks von Akteuren sind, in dem die EPA eine zwar wichtige aber nicht allein entscheidende Rolle spielt. Die EPA muss bei der Entwicklung ihrer Ziele die Einflüsse andere Akteure mit einbeziehen. Diese können bei der Entwicklung und der Erreichung von realistischen und geeigneten Performancemaßstäben eine wesentliche Rolle spielen.

- Langfristigkeit von Umweltwirkungen: Bedeutsame Veränderungen der Umweltsituation werden vielfach erst über lange Fristen manifest. Demzufolge sind die Ergebnisse der EPA möglicherweise nicht klar als jährlicher Outcome für die Umwelt identifizierbar. Dementsprechend besteht die Herausforderung diese Maßnahme-Wirkungsbeziehungen so weit als möglich abschätzbar zu machen.

- Kostenrechnungssysteme müssen notwendigerweise in Bezug gesetzt werden zu der spezifischen „mission“. Daher ist es bereits frühzeitig erforderlich, dass die wesentlichen Verantwortlichkeitssegmente auch zum Zwecke der Kostenzurechnung definiert werden. Dabei besteht die Notwendigkeit,

- die Budgetstrukturen der Behörden so zu verändern, dass auf Programme und Outcome fokussiert wird,

- das Problem der Zuordnung der Ergebnisse und der Finanzierung zu lösen, das sich aus überlappenden Programmen ergibt, und

- die Gemeinkosten auf unterschiedlichste Projekte, Programme und Verantwortungszentren zu verteilen.

\section{Fazit}

Der amerikanische Reformansatz ist interessant, da die jeweiligen Verwaltungseinheiten (auch die EPA) ihre grundsätzlichen und langfristigen Ziele definieren mussten. Dies ist keineswegs selbstverständlich. Vor allen Dingen mussten sie auch Wege aufzeigen, wie diese Ziele mit den zur Verfügung stehenden Mitteln und in Kooperation mit den unterschiedlichen Akteuren erreicht werden können. Damit wird die Transparenz des Verwaltungshandelns und der Zielorientierung deutlich erhöht, es kann nachvollzogen werden was mit welchen Maßnahmen erreicht werden soll.

Die Diskussion um Umweltziele ist auch in Deutschland durchaus nichts Neues - abgesehen davon dass es noch (fast) keine gibt-, aber die konkrete Verknüpfung von Verwaltungshandeln und Budget- zuordnungen ist eine neue Orientierung, die deutlich über die Ansätze der Verwaltungsreform in Deutschland hinausgeht. Beispielsweise war in Berlin die Verwaltungsreform auf den Output orientiert, eine enge Verknüpfung etwa mit Nachhaltigkeitszielen (Outcome) hat nicht stattgefunden. Insofern werden Aktivitäten gemessen aber nicht die Verknüpfung mit den eigentlichen Zielen des Verwaltungshandelns.

Und noch etwas wurde deutlich: Diese Herangehensweise verhindert den Bau von Luftschlössern. Die Aufstellung von Nachhaltigkeitszielen muss nach dieser Verfahrensweise entsprechend unterlegt werden. Es müssen die Schritte und Wege aufgezeigt werden, wie und in Kooperation mit welchen Akteuren dieses Ziel auch erreicht werden kann. Damit würde eine Verbindung zwischen politischer Deklamation und der realen Umsetzung hergestellt werden können, die entweder zur Folge hat, dass nicht erreichbare Ziele auch nicht aufgestellt werden oder - um die Glaubwürdigkeit nicht zu verlieren - entsprechende Aktionspläne aufgestellt und mit finanziellen Mitteln unterlegt werden.

In der Summe erweist sich daher der schleswigholsteinische Ansatz als hochinteressant und hat noch eine Vielzahl komplexer Fragestellungen zu lösen: von der Frage der Indikatoren, über die Frage der Kosten- und Leistungsrechnung, den Zuschnitt der Verwaltung bis hin zur Frage der Kooperation mit anderen für den Umweltbereich wichtigen Akteuren, etwa anderen Ministerien, den Unternehmen und nicht zuletzt der Bevölkerung. Das Verständnis von Verwaltungshandeln könnte sich insofern umfassend verändern.

\section{Anmerkungen}

(1) Vgl. Ökologie-Zentrum Kiel/ IÖW: Entwicklung eines Indikatorensystems für ein strategisches Steuerungssystem. Unveröffentlichter Endbericht. Kontakt für weitere Infos: ÖZK Wilhelm Windhorst, E-mail: wilhelm@pz-oekosys.uni-kiel.de. Das IÖW hatte die Aufgabe, die Möglichkeiten der Verknüpfung der Indikatoren mit der KLR und dem Controlling zu überprüfen.

(2) Vgl. für nähere Informationen im Internet unter http:// www.epa.gov/epahome/aboutepa.htm

\section{Die Autorlnnen}

Susanne Donner ist freie wissenschaftliche Mitarbeiterin, Ulrich Petschow ist wissenschaftlicher Mitarbeiter am IÖW; Felicitas Kubala ist Leiterin des Umweltamtes Berlin-Steglitz.

Kontakt: IÖW, Potsdamer Str. 105, 10785 Berlin. Tel. 030/ 884594-0, Fax 030/ 8825439, E-mail: Ulrich.Petschow@ioew.de 
(c) 20I0 Authors; licensee IÖW and oekom verlag. This is an article distributed under the terms of the Creative Commons Attribution Non-Commercial No Derivates License (http://creativecommons.org/licenses/by-nc-nd/3.o/), which permits unrestricted use, distribution, and reproduction in any medium, provided the original work is properly cited. 